\title{
Local intra-articular injection of rapamycin delays articular cartilage degeneration in a murine model of osteoarthritis
}

Koji Takayama ${ }^{1,2}$, Yohei Kawakami 1,2, Makoto Kobayashi ${ }^{1,2}$, Nick Greco ${ }^{1,2}$, James H Cummins ${ }^{2}$, Takehiko Matsushita ${ }^{3}$, Ryosuke Kuroda ${ }^{3}$, Masahiro Kurosaka ${ }^{3}$, Freddie $\mathrm{H} \mathrm{Fu}^{1,2}$ and Johnny Huard ${ }^{1,2,4^{*}}$

\begin{abstract}
Introduction: Recent studies have revealed that rapamycin activates autophagy in human chondrocytes preventing the development of osteoarthritis (OA) like changes in vitro, while the systemic injection of rapamycin reduces the severity of experimental osteoarthritis in a murine model of OA in vivo. Since the systemic use of rapamycin is associated with numerous side effects, the goal of the current study was to examine the beneficial effect of local intra-articular injection of rapamycin in a murine model of $\mathrm{OA}$ and to elucidate the mechanism of action of rapamycin on articular cartilage.
\end{abstract}

Methods: Destabilization of the medial meniscus (DMM) was performed on 10-week-old male mice to induce OA. Intra-articular injections of $10 \mu \mathrm{l}$ of rapamycin $(10 \mu \mathrm{M})$ were administered twice weekly for 8 weeks. Articular cartilage damage was analyzed by histology using a semi-quantitative scoring system at 8 and 12 weeks after surgery.

Mammalian target of rapamycin (mTOR), light chain 3 (LC3), vascular endothelial growth factor (VEGF), collagen, type X alpha 1 (COL10A1), and matrix metallopeptidase 13 (MMP13) expressions were analyzed by immunohistochemistry. VEGF, COL10A1, and MMP13 expressions were further examined via quantitative RT-PCR (qPCR).

Results: Intra-articular injection of rapamycin significantly reduced the severity of articular cartilage degradation at 8 and 12 weeks after DMM surgery. A reduction in mTOR expression and the activation of LC3 (an autophagy marker) in the chondrocytes was observed in the rapamycin treated mice. Rapamycin treatment also reduced VEGF, COL10A1, and MMP13 expressions at 8 and 12 weeks after DMM surgery.

Conclusion: These results demonstrate that the intra-articular injection of rapamycin could reduce mTOR expression, leading to a delay in articular cartilage degradation in our OA murine model. Our observations suggest that local intra-articular injection of rapamycin could represent a potential therapeutic approach to prevent OA.

\section{Introduction}

Osteoarthritis $(\mathrm{OA})$ is the most common disorder of the joint, causing joint pain and dysfunction in affected patients. Multiple factors have been suggested in the pathogenesis of $\mathrm{OA}$, including mechanical, genetic, and aging-associated factors. At the cellular level, $\mathrm{OA}$ is characterized by a loss of tissue cellularity and extracellular matrix (ECM) damage [1]. Chondrocytes are the

\footnotetext{
*Correspondence: jhuard@pitt.edu

'Stem Cell Research Center, University of Pittsburgh, Pittsburgh, PA 15219, USA

${ }^{2}$ Department of Orthopaedic Surgery, University of Pittsburgh, Pittsburgh, PA 15260, USA

Full list of author information is available at the end of the article
}

resident cells found in cartilage tissue and are responsible for both synthesis and turnover of the ECM [2]; therefore, maintaining the health of chondrocytes is an important factor for preventing articular cartilage degeneration.

Autophagy is a cellular self-digestion process which is evolutionally observed among species and generally activated under conditions of nutrient deprivation. When cells experience nutrient deprivation, they maintain only the minimum amount of essential components in order to prevent energy loss, thereby degrading unnecessary intracellular components through the process of autophagy. Thus, autophagy is perceived as an important mechanism for cell survival when exposed to various stresses [3]. Recently, a growing number of studies have revealed 
that autophagy also plays an important role in physiological housekeeping processes through the intracellular clearance of unnecessary proteins, pathogens, and damaged organelles, which include mitochondria, peroxisomes, and the endoplasmic reticulum (ER) [4-6]. These functions have important implications in the pathogenesis of a variety of human diseases, such as neurodegenerative, cardiac, muscular, and inflammatory diseases [4]. One of the common pathological discoveries in such diseases is the accumulation of aggregate-prone proteins, potentially harmful to the cells, which likely occur due to an impairment of autophagy [7]. Autophagy has, therefore, been suggested to play a preventive role in the development of particular human diseases. Based on the broad cell-protective functions of autophagy, it is plausible that autophagy play a protective role in chondrocytes under stresses, and may prevent the OA degeneration of chondrocytes.

Recent studies have revealed that rapamycin activates autophagy in human chondrocytes in vitro, which acts to prevent the expression of OA-like changes [8]. Similarly, the systemic injection of rapamycin has been shown to reduce the severity of OA in an experimental murine model [9]. Clinically, rapamycin is currently used as an immunosuppressant in organ transplantation patients. Clinical studies have shown that rapamycin is not only able to provide a low rate of acute rejection and significant improvement in kidney allograft function, but it can also significantly decrease the incidence of posttransplant malignancies [10,11]; however, the systemic use of rapamycin is associated with many side effects, including increases in serum cholesterol and triglycerides, anemia, proteinuria, skin rashes, delayed wound healing, and diarrhea, which can eventually lead to rapamycin withdrawal [12].

Due to these severe side effects, the local intra-articular injection of rapamycin may be more appropriate for clinical use than systemic administration. However, it is unknown whether the intra-articular injection of rapamycin can affect the degeneration of articular cartilage. The objective of this study was to examine the effect of local intra-articular injection of rapamycin on articular cartilage in a murine model of OA. Our observations suggest that intra-articular injection of rapamycin protects articular cartilage from osteoarthritic changes, and may represent an effective and safe therapeutic delivery method to prevent articular cartilage degeneration.

\section{Methods}

\section{Surgical induction of osteoarthritis in mice}

All studies were performed according to protocols approved by the University of Pittsburgh's Institutional Animal Care and Use Committee. Forty, 10-week-old, male $\mathrm{C} 57 \mathrm{Bl} / 6 \mathrm{~J}$ mice were used in this study. The animals were anesthetized with $3 \%$ isoflurane in $\mathrm{O}_{2}$ gas (1.5 liter/minute). Experimental osteoarthritis was induced by destabilizing the medial meniscus (DMM) in the right knee [13]. Briefly, the joint capsule was opened with an incision just medial to the patellar tendon and the medial meniscotibial ligament was sectioned with microsurgical scissors. As a control, surgery was performed on left knee joints but the ligaments were left intact and used as sham joints. All mice were allowed to move freely within their cages after surgery. After surgical induction of $\mathrm{OA}$, the animals were divided into two groups, namely the rapamycin treatment group and the dimethyl sulphoxide (DMSO) (control) group. Mice were sacrificed at 8 and 12 weeks after DMM surgery and subjected to histological and gene expression analyses.

\section{Rapamycin treatment}

To perform the intra-articular injection, the animals were first anesthetized and the skin was subsequently incised longitudinally over the center of the knee joint. The capsule and patellar tendon were exposed to clarify the anatomy of the knee to ensure reproducible intraarticular injection of either rapamycin or DMSO (control). Rapamycin was obtained from LC Laboratories (Woburn, MA, USA) and was dissolved in dimethyl sulphoxide (DMSO) to make a $50 \mathrm{mg} / \mathrm{ml}$ stock solution. For injection, the stock solution was diluted in PBS: $10 \mu \mathrm{l}$ of rapamycin $(10 \mu \mathrm{M})$ with DMSO $(0.02 \%)$ was administered twice a week for 8 weeks in both knees in the rapamycin group. The dosage and frequency of rapamycin was selected based on previous studies $[8,14]$. These studies have demonstrated that autophagy activation by $10 \mu \mathrm{M}$ rapamycin regulated the changes in the expression of OA-related genes through the modulation of apoptosis and reactive oxygen species (ROS) in human chondrocytes in vitro [8], and the effect of rapamycin on mechanical-injury-induced cell death continued up to 96 hours after treatment in ex vivo study [14]. The control group received intra-articular injections of $10 \mu \mathrm{l}$ of DMSO $(0.02 \%)$ in both knees according to the same schedule as the rapamycin group.

\section{Histological evaluation for articular cartilage degeneration}

Six knee joints from each group were fixed in $10 \%$ neutral buffered formalin, decalcified with $10 \%$ formic acid, and embedded in paraffin. Coronal histological sections were performed through the joint at $80-\mu \mathrm{m}$ intervals and stained with toluidine blue, and articular cartilage damage was scored by two observers blinded to sample identity using a scoring system reported by Glasson [15]. In this system, histological scores were measured in four quadrants (medial femoral condyle, medial tibial plateau, lateral femoral condyle, and lateral tibial plateau). The scores are defined as follows: 0: normal; 0.5: loss of 
toluidine blue without structural changes; 1 : small fibrillations without loss of cartilage; 2: vertical clefts extending from the articular surface down to the layer immediately below the superficial tangential zone with some loss of surface lamina; 3: vertical clefts/erosion extending down to the calcified articular cartilage comprising $<25 \%$ of the quadrant width; 4: vertical clefts/erosion extending down to the articular calcified cartilage comprising 25 to $50 \%$ of the quadrant width; 5: vertical clefts/erosion extending down to the calcified articular cartilage comprising 50 to $75 \%$ of the quadrant; and 6: vertical clefts/erosion extending down to the calcified articular cartilage comprising $>75 \%$ of the quadrant width. Ten sections of each knee joint were scored and the final score was expressed as the summed histologic score for each joint. The summed score represents the additive score of each quadrant of the joint on each histologic section through the joint. This method of analysis enabled assessment of the severity of the lesions while also reflecting the surface area of articular cartilage affected with OA lesions.

\section{Immunohistochemistry}

We performed immunohistochemical staining to detect the expression of phosphor-mammalian target of rapamycin (p-mTOR), light chain 3 (LC3), vascular endothelial growth factor (VEGF), collagen, type $\mathrm{X}$ alpha 1 (COL10A1), and matrix metallopeptidase 13 (MMP13) in the articular cartilage. Rabbit polyclonal antibodies to p-mTOR, LC3 (Cell Signaling, Beverly, MA, USA), VEGF, MMP13 (Abcam, Cambridge, MA, USA), and COL10A1 (Abbiotec, San Diego, CA, USA) were used at a 1:100 dilution and incubated overnight at $4^{\circ} \mathrm{C}$. Alexa Fluor 488-conjugated donkey anti-rabbit IgG (Molecular Probes, Grand Island, NY, USA) was used at 1:200 dilution as the secondary antibody against the primary antibodies at room temperature for 2 hours. After staining, we evaluated the expression levels in the articular cartilage using Northern Eclipse software (Empix Imaging Inc, Cheektowaga, NY, USA).

\section{RNA isolation and quantitative RT-PCR (qPCR)}

Four knee joint specimens from both the control and experimental group were used for qPCR. Total RNA was extracted from cartilage using TRIzol reagent (Invitrogen, Carlsbad, CA, USA) with QIAshredder homogenizers and the RNeasy Mini kit (Qiagen, Hilden, Germanay) according to the manufacturer's protocol. One microgram of total RNA was used for random hexamer-primed cDNA synthesis using the SuperScript II pre-amplification system (Invitrogen, Carlsbad, CA,USA). Quantitative RT-PCR reactions were performed in triplicate using iQ5 (Bio-Rad, Hercules, CA, USA) with Maxima SYBR Green/ROX qPCR Master Mix (Thermo, Thermo Scientific, Rockford, IL, USA) and $300 \mathrm{nM}$ of each primer. The primers were designed based on the sequences in the GenBank database. The primer pairs used for this study are shown in Table 1. Specificity of the reactions was confirmed by $2.5 \%$ agarose gel electrophoresis.

\section{Statistical analysis}

All the data were expressed as the mean $\pm \mathrm{SD}$. The MannWhitney $U$-test was used for direct comparisons between the two groups. One-way analysis of variance (ANOVA) or the Kruskal-Wallis test was applied for multiple comparisons between independent groups. Pairwise, multiple comparisons were performed using the Tukey-Kramer or Scheffé post hoc test. Data analyses were performed using PASW Statistics 21 (SPSS, Chicago, IL, USA). Statistical significance was determined at level of $P<0.05$.

\section{Results}

The local intra-articular injection of rapamycin delayed articular cartilage degradation

There were no structural changes at the anterior cruciate ligament or meniscus identified in the sham knees that were treated with rapamycin or DMSO. Side effects such as weight loss, skin rashes, delayed wound healing, or diarrhea were not observed after local intra-articular injection of rapamycin. Histological sections demonstrated significantly less articular cartilage degeneration in the experimental group treated with local intra-articular injections of rapamycin at 8 and 12 weeks after induction of $\mathrm{OA}$ with DMM surgery compared to the OA-induced mice treated with DMSO. Histological grading showed that the DMSO-treated mice had a loss of proteoglycan staining with articular fibrillation at 8 weeks and losses of hyaline cartilage, proteoglycan staining, and lesions extending into the calcified cartilage at 12 weeks after surgery (Figure 1A). In contrast, rapamycin-treated mice showed a focal loss of proteoglycan staining without severe articular cartilage loss at 8 weeks and lesions with a loss of proteoglycan staining slightly increased 12 weeks after surgery; however, hyaline cartilage was preserved (Figure 1B). The extent of OA was evaluated by scoring specific parameters of OA, and is presented as summed scores (the higher the score

\begin{tabular}{|c|c|c|c|}
\hline Gene & & 5' DNA sequence $3^{\prime}$ & $\begin{array}{l}\text { Amplicon } \\
\text { length, } \\
\text { base pairs }\end{array}$ \\
\hline \multirow[t]{2}{*}{ VEGF } & Forward & CCCACGTCAGAGAGCAACA & 101 \\
\hline & Reverse & TCACATCTGCTGTGCTGTAGG & \\
\hline \multirow[t]{2}{*}{ Col10 } & Forward & AGGCAAGCCAGGCTATGGAA & 83 \\
\hline & Reverse & GCTTCCCCGTGGCTGATATTC & \\
\hline \multirow[t]{2}{*}{ MMP13 } & Forward & GCTGCGGTTCACTITGAGAA & 106 \\
\hline & Reverse & GGCGGGGATAATCTTTGTCCA & \\
\hline
\end{tabular}




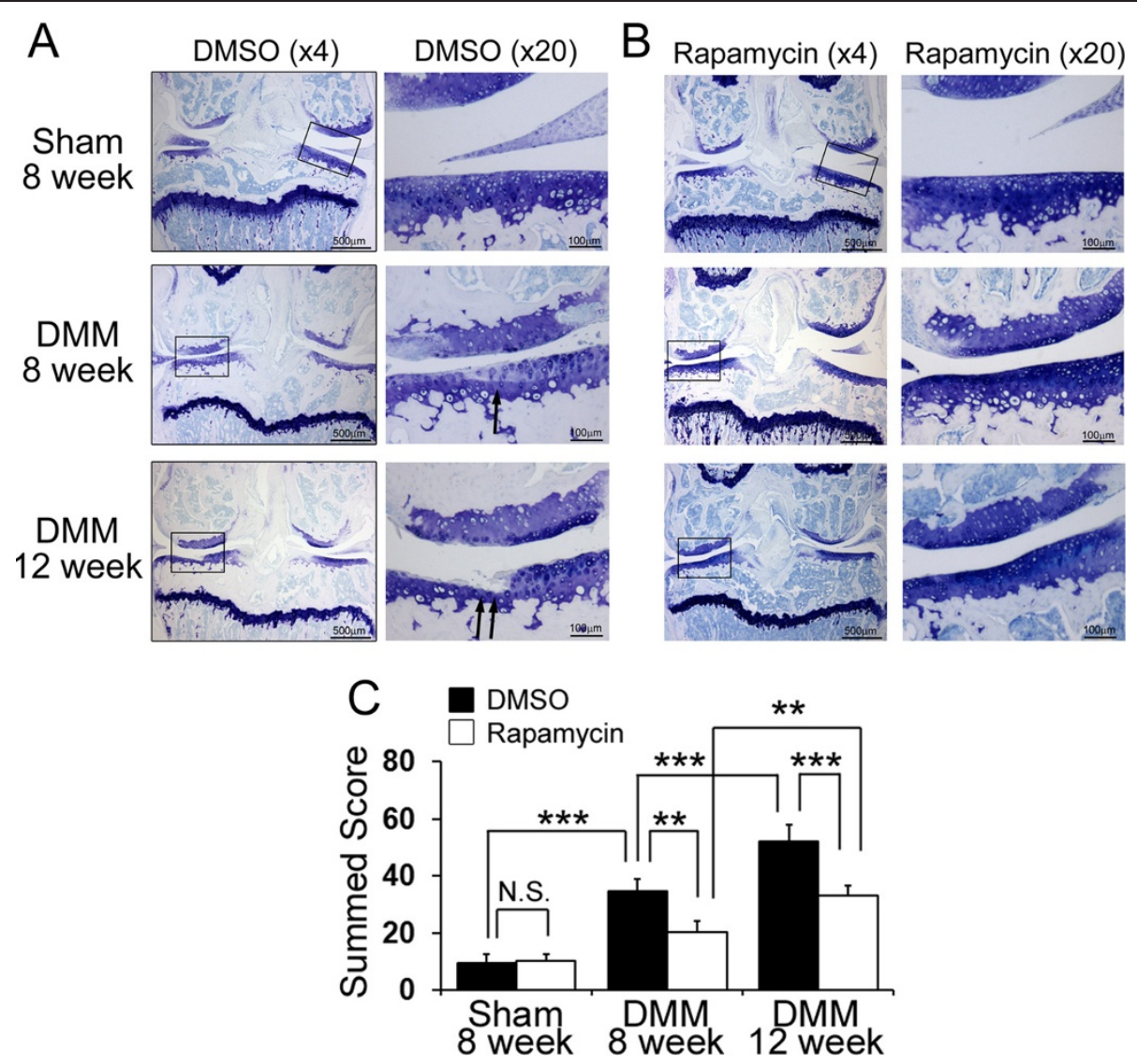

Figure 1 Histological evaluation of osteoarthritis (OA). (A, B) Representative images of toluidine blue staining from dimethyl sulphoxide (DMSO) (A) and rapamycin (B) treated mice at 8 and 12 weeks after destabilization of the medial meniscus (DMM) or sham surgery. Boxed areas in the toluidine blue stained image at the left side $(x 4)$ indicate the regions shown in the enlarged toluidine blue stained area at the right side (x20). Scale bar $=500 \mu \mathrm{m}(\mathrm{x} 4)$ and $100 \mu \mathrm{m}(\mathrm{x} 10)$. Arrows indicate the osteoarthritic change after DMM surgery. (C) Graph indicating the summed OA scores. Summed OA scores were calculated from all four quadrants and eight sections from each knee. ${ }^{* * *} P<0.001,{ }^{* *} P<0.01$.

the greater the articular cartilage degeneration) (Figure 1C, Table 2). Using the summed OA score, DMSO-treated mice developed $\mathrm{OA}$ in a time-dependent manner and had a significantly higher score than the rapamycintreated mice at 8 and 12 weeks following DMM surgery (Figure $1 \mathrm{C}, P=0.001$ at 8 weeks and $P<0.001$ at 12 weeks). The summed OA score in the rapamycintreated mice at 12 weeks was increased compared to the score at 8 weeks (Figure $1 C, P<0.001$ ). These results suggested that the intra-articular injection of rapamycin did

Table 2 Summed score

\begin{tabular}{lll}
\hline Summed score & & \\
\hline Sham 8 weeks & DMSO & 9.7 (SD 2.9) \\
& Rapamycin & 10.2 (SD 2.5) \\
DMM 8 weeks & DMSO & 34.7 (SD 4.5) \\
& Rapamycin & 20.5 (SD 3.6) \\
DMM 12 weeks & DMSO & 52.2 (SD 6.0) \\
& Rapamycin & 33.3 (SD 3.3) \\
\hline
\end{tabular}

DMM, destabilization of the medial meniscus; DMSO, dimethyl sulphoxide. not completely prevent, but delayed articular cartilage degradation in the presence of meniscus injury after DMM surgery.

The local intra-articular injection of rapamycin decreased p-mTOR and increased LC3 expression

To determine whether the local intra-articular injection of rapamycin modulates mTOR and autophagy, the effects that rapamycin had on phospho-mTOR (p-mTOR) and LC3 expressions were examined. The expression of p-mTOR was increased in DMSO-treated mice at 8 and 12 weeks post DMM surgery compared to DMSOtreated mice undergoing the sham knee operation. Rapamycin treatment suppressed p-mTOR expression in these mice compared to those treated with DMSO at both the 8- and 12-week time points post DMM injury. The expression of p-mTOR in the rapamycin-treated mice was significantly increased at 12 weeks compared to 8 weeks after DMM injury (at 8 weeks DMSO $108.5 \pm$ 13.3 , rapamycin $43.0 \pm 8.9$; at 12 weeks DMSO $273.8 \pm$ 27.0, rapamycin $76.5 \pm 14.7$ ) (Figure $2 \mathrm{~A}, \mathrm{~B}$ ). In contrast, 


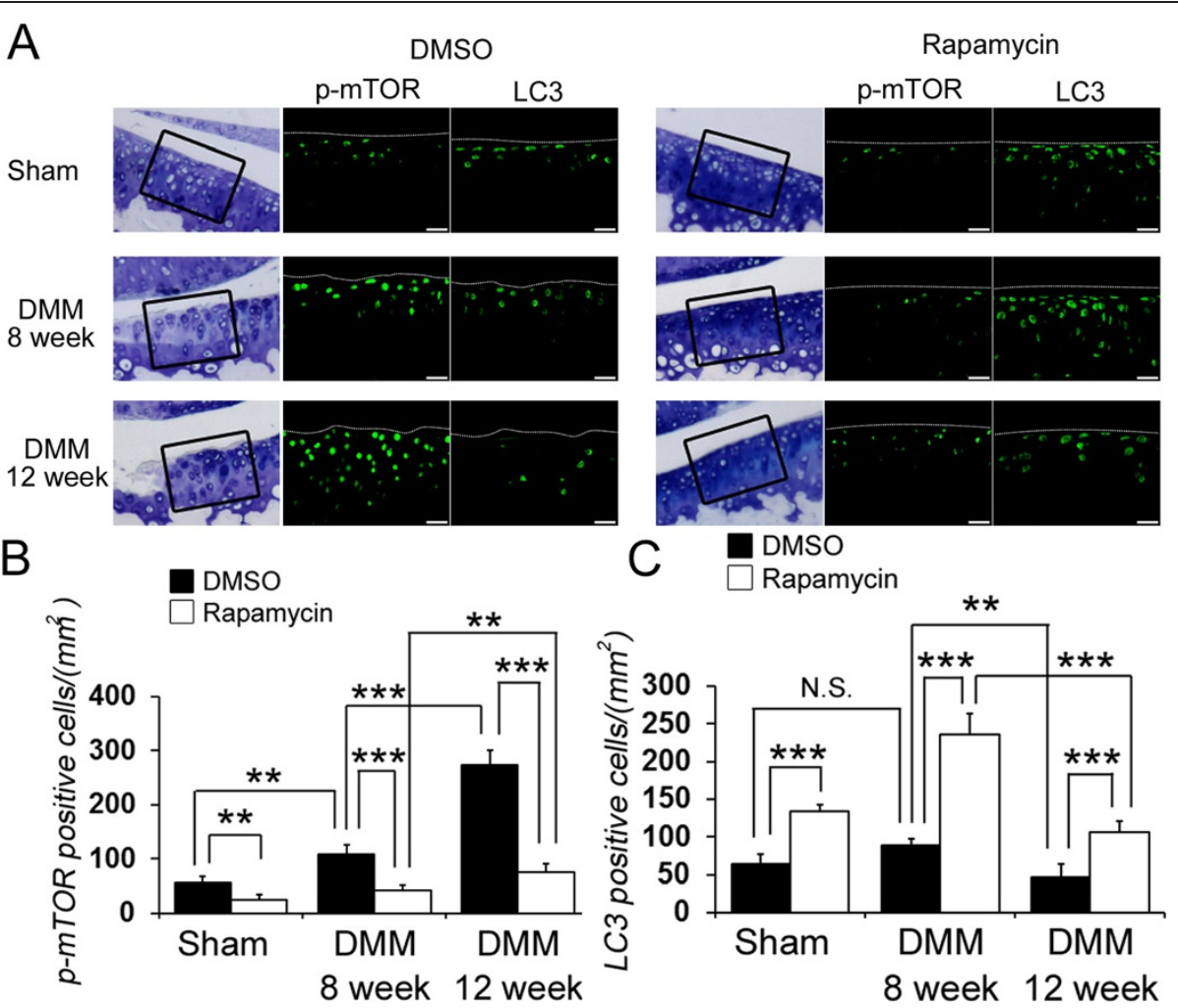

Figure 2 The effect of rapamycin on phospho- mammalian target of rapamycin (p-mTOR) and light chain 3 (LC3) expression.

(A) Representative images of immunostaining for p-mTOR (green) and LC3 (green). Scale bar = $20 \mu \mathrm{m}$. (B) Quantification of p-mTOR-positive cells was calculated. Rapamycin treatment suppressed p-mTOR expression in the rapamycin-treated mice compared to those treated with dimethyl sulphoxid (DMSO) at both the 8- and 12-week time points (at 8 weeks DMSO 108.5 \pm 13.3 , rapamycin $43.0 \pm 8.9 \mathrm{~mm}$; at 12 weeks DMSO 273.8 \pm 27.0 , rapamycin $76.5 \pm 14.7 \mathrm{~mm}$ ). (C) Quantification of LC3-positive cells was calculated. ${ }^{* *} P<0.01$, ${ }^{* *} P<0.001$. DMM, destabilization of the medial meniscus.

there were more LC3-positive cells in the articular cartilage maintaining proteoglycan staining but fewer in the degenerated articular cartilage of the DMSO-treated mice after DMM surgery. An increase in LC3-positive cells was observed in the rapamycin-treated mice compared to those treated with DMSO at 8 and 12 weeks post DMM surgery. The number of LC3-positive cells in the rapamycin-treated mice decreased significantly at 12 weeks compared to 8 weeks post DMM injury (at 8 weeks DMSO 88.2 \pm 13.1 , rapamycin $236.0 \pm 56.3$; at 12 weeks DMSO $46.2 \pm 12.8$, rapamycin $106.3 \pm 27.1$ ) (Figure $2 \mathrm{~A}, \mathrm{C}$ ).

The intra-articular injection of rapamycin decreased VEGF expression in articular cartilage

As it has been reported that the expression of VEGF is related to the development of OA changes [16], VEGF expression was examined with immunohistochemistry and qPCR (Figure 3). The number of VEGF-positive cells in the articular cartilage was significantly lower in the rapamycin group compared with the DMSO group at 8 and 12 week post DMM injury (at 8 weeks DMSO $143.8 \pm 29.6$, rapamycin $21.2 \pm 8.4, P<0.001$ for DMSO versus rapamcyin; at 12 weeks DMSO 275.5 \pm 56.9 , rapamycin $96.0 \pm 27.8, P<0.001$ for DMSO versus rapamcyin). The number of VEGF-positive cells in the rapamycintreated mice increased at 12 weeks compared to 8 weeks post DMM injury $(P<0.001$ for DMSO at 8 weeks versus 12 weeks) (Figure 3A, B). Similarly, the expression of VEGF by qPCR revealed a significant increase in the DMSOtreated mice at 8 weeks after DMM surgery compared to sham knees (Figure 3C, $P<0.001$ relative to sham). Rapamycin treatment decreased VEGF expression when compared to the DMSO-treated mice at the 8 and 12 week time points post DMM injury (Figure $3 \mathrm{C}, P<0.001$ at 8 and 12 weeks), suggesting that the local intra-articular injection of rapamycin reduced articular cartilage damage, at least in part, through a reduction in VEGF expression.

The local intra-articular injection of rapamycin decreased CoL10A1 and MMP13 expression

To investigate the mechanism behind rapamycin's ability to prevent experimental $\mathrm{OA}$, subsequent experimentation focused on chondrocyte hypertrophy and consequently the expression of COL10A1 and MMP13 (hypertrophic chondrocyte markers) was examined by immunohistochemistry and qPCR (Figures 4, 5). The number 


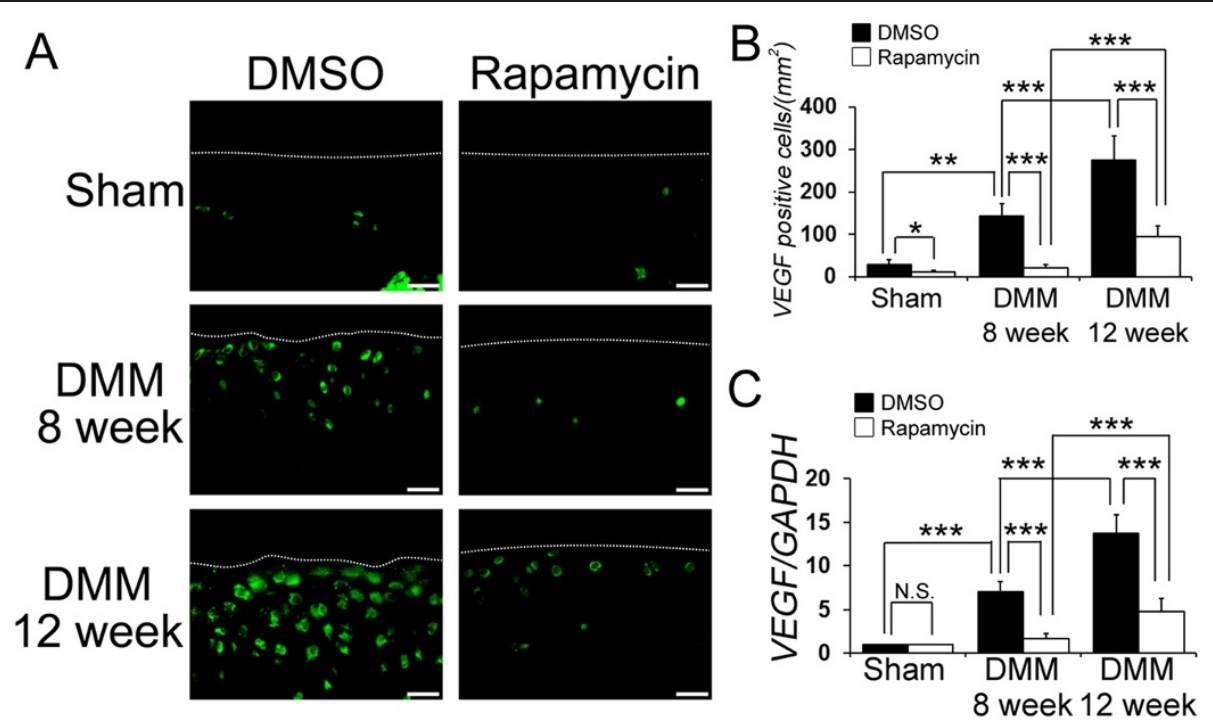

Figure 3 The effect of rapamycin on vascular endothelial growth factor (VEGF) expression in the articular cartilage. (A) Representative images of immunostaining for VEGF (green). Scale bar $=20 \mu \mathrm{m}$. (B) Quantification of VEGF-positive cells in the cartilage was calculated. (C) Graph indicating quantitative RT-PCR for VEGF in dimethyl sulphoxide (DMSO)- and rapamycin-treated mice at 8 and 12 weeks after destabilization of the medial meniscus (DMM) or sham surgery. ${ }^{*} P<0.05,{ }^{* *} P<0.01$, ${ }^{* * *} P<0.001$.

of CoL10A1-positive cells in the articular cartilage was significantly lower in the rapamycin group when compared to the DMSO group at 8 and 12 weeks post DMM injury (at 8 weeks DMSO 88.2 \pm 13.1 , rapamycin $236.0 \pm 56.3$, $P<0.001$ for DMSO versus rapamcyin; at 12 weeks DMSO 46.2 \pm 12.8 , rapamycin $106.3 \pm 27.1, P<0.001$ for DMSO versus rapamcyin). The number of CoL10A1positive cells in the rapamycin-treated mice increased at
12 weeks compared to 8 weeks post DMM injury $(P<0.001$ for DMSO at 8 weeks versus 12 weeks) (Figure 4A, B). Similarly the expression of COL10A1, as measured by qPCR, was increased in the DMSO-treated mice at 8 and 12 weeks after DMM surgery compared to the sham knees (Figure 4C: $P=0.006$ at 8 weeks, $P<0.001$ at 12 weeks, respectively). Also intra-articular injection of rapamycin significantly decreased the expression of COL10A1 compared

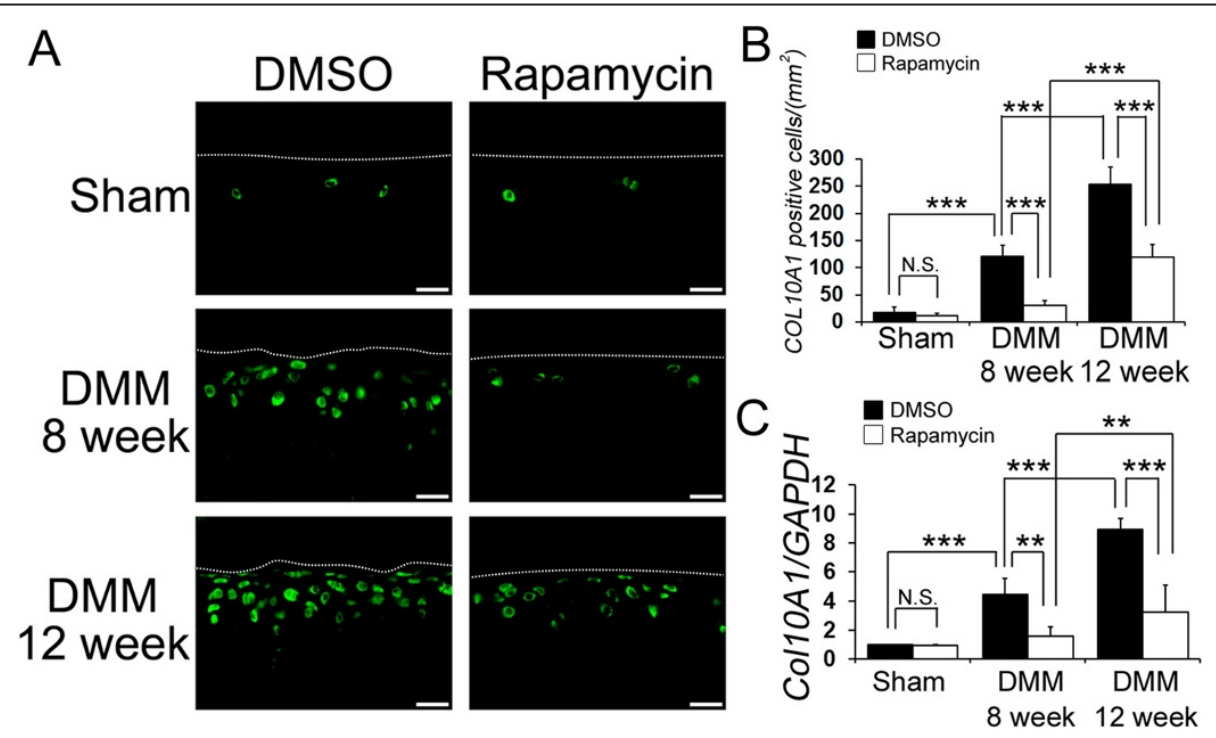

Figure 4 The effect of rapamycin on collagen, type $X$ alpha 1 (COL10A1) expression in articular cartilage. (A) Representative images of immunostaining for COL10A1 (green). Scale bar $=20 \mu \mathrm{m}$. (B) Quantification of COL10A1-positive cells in the cartilage was calculated. (C) Graph indicating quantitative RT-PCR for COL10A1 in dimethyl sulphoxide (DMSO)- and rapamycin-treated mice 8 and 12 weeks after destabilization of the medial meniscus (DMM) injury. ${ }^{* *} P<0.001,{ }^{* *} P<0.01$. 


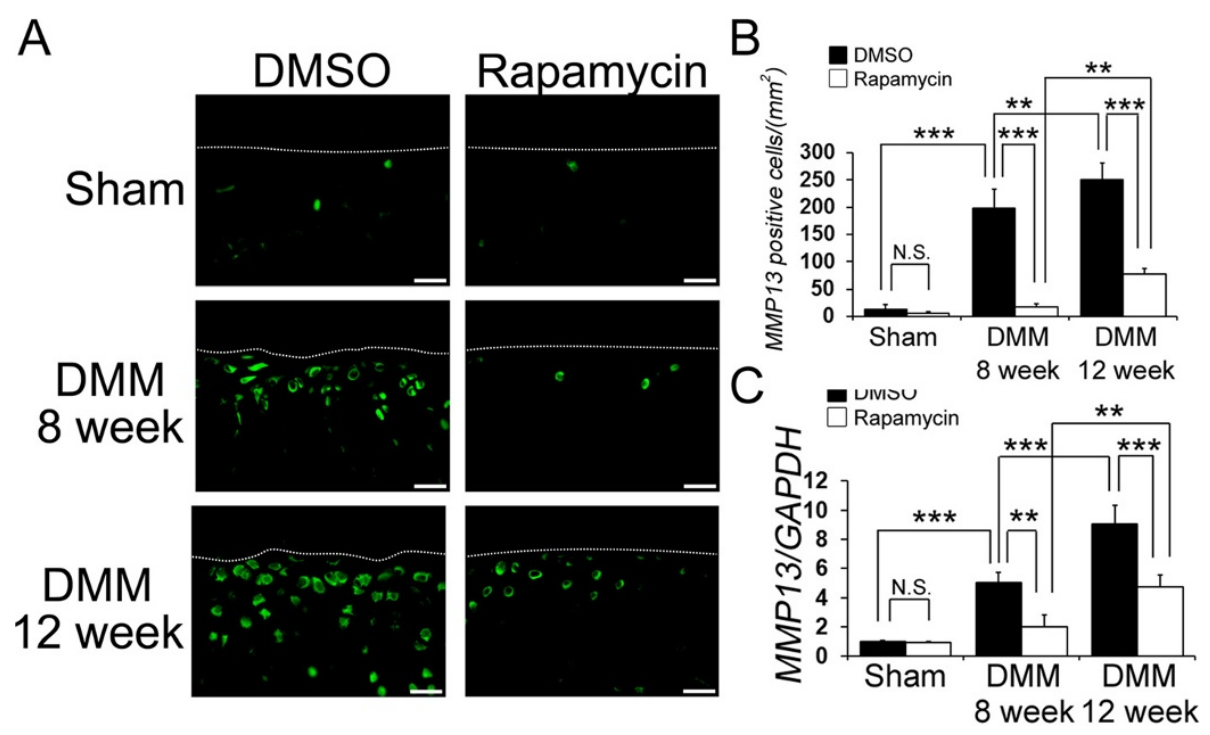

Figure 5 The effect of rapamycin on matrix metallopeptidase 13 (MMP13) expression in articular cartilage. (A) Representative images of immunostaining for MMP13 (green). Scale bar $=20 \mu \mathrm{m}$. (B) Quantification of MMP13-positive cells in the cartilage was calculated. (C) Graph indicating quantitative RT-PCR for MMP13 in dimethyl sulphoxide (DMSO)- and rapamycin-treated mice 8 and 12 weeks after destabilization of the medial meniscus (DMM) injury. ${ }^{* *} P<0.001,{ }^{* *} P<0.01$

to the DMSO-treated mice at 8 and 12 weeks after DMM surgery (Figure 4C: $P=0.001$ at 8 weeks, $P<0.001$ at 12 weeks, respectively).

The number of MMP13-positive cells in the cartilage was significantly lower in the rapamycin group compared with the DMSO group at 8 and 12 weeks post DMM injury (at 8 weeks DMSO $198.2 \pm 34.6$, rapamycin $18.0 \pm 5.7, P<0.001$ for DMSO versus rapamcyin; at 12 weeks DMSO $249.8 \pm 31.1$, rapamycin $77.8 \pm 10.0$, $P<0.001$ for DMSO versus rapamcyin). The number of MMP13-positive cells in the rapamycin-treated mice increased at 12 weeks compared to 8 weeks post DMM injury $(P<0.01$ for DMSO at 8 weeks versus 12 weeks) (Figure 5A, B). Rapamycin treatment reduced MMP13 expression, as measured by qPCR, at 8 and 12 weeks after DMM surgery compared to the MMP13 expression in the DMSO-treated mice (Figure $5 \mathrm{C}, P=0.026$ at 8 weeks, $P<0.001$ at 12 weeks). These results suggest that mTOR inhibition with rapamycin decreases hypertrophic change in articular cartilage after DMM surgery.

\section{Discussion}

The results of the current study are the first to demonstrate that articular cartilage degeneration occurring after DMM surgery correlates with increased in mTOR expression, and consequently local intra-articular injection of rapamycin reduced mTOR expression, which delayed articular cartilage degradation. Recently, systemic injection of rapamycin has been shown to reduce the severity of $\mathrm{OA}$ in an experimental murine model [9]. The systemic use of rapamycin in the clinical setting is associated with many side effects, such as weight loss, skin rashes, delayed wound healing, or diarrhea, which can eventually lead to rapamycin withdrawal [12]. In this study, these side effects were not observed after local intra-articular injection of rapamycin. The local intraarticular injection of rapamycin might be more appropriate for clinical use than systemic administration.

In this study, we demonstrated that after surgically inducing joint instability, chondrocytes in the articular cartilage displayed increased mTOR expression during the progression of OA, suggesting that the activation of mTOR leads to articular cartilage degeneration. Furthermore, the inhibition of mTOR with rapamycin promoted a significant delay in the progression of OA changes, as demonstrated histologically by staining the AC for proteoglycan content.

Recent studies have demonstrated that mTOR inhibition by rapamycin triggers a negative feedback loop, resulting in the activation of Akt signaling $[17,18]$. In adult articular cartilage, phosphoinositide 3 (PI-3) kinase-Akt signaling promotes matrix synthesis as well as the survival of chondrocytes. Activation of Akt in human articular chondrocytes significantly increase proteoglycan synthesis and type II collagen expression [19-21] (Figure 6). Another important function of the mTOR signaling pathway is the regulation of autophagy, a process in which the cell degrades damaged or excess cellular components, ranging from individual proteins and protein aggregates to whole organelles, through the use of the cell lysosomal machinery. A previous in vitro study indicated that autophagy activation by $10 \mu \mathrm{M}$ 


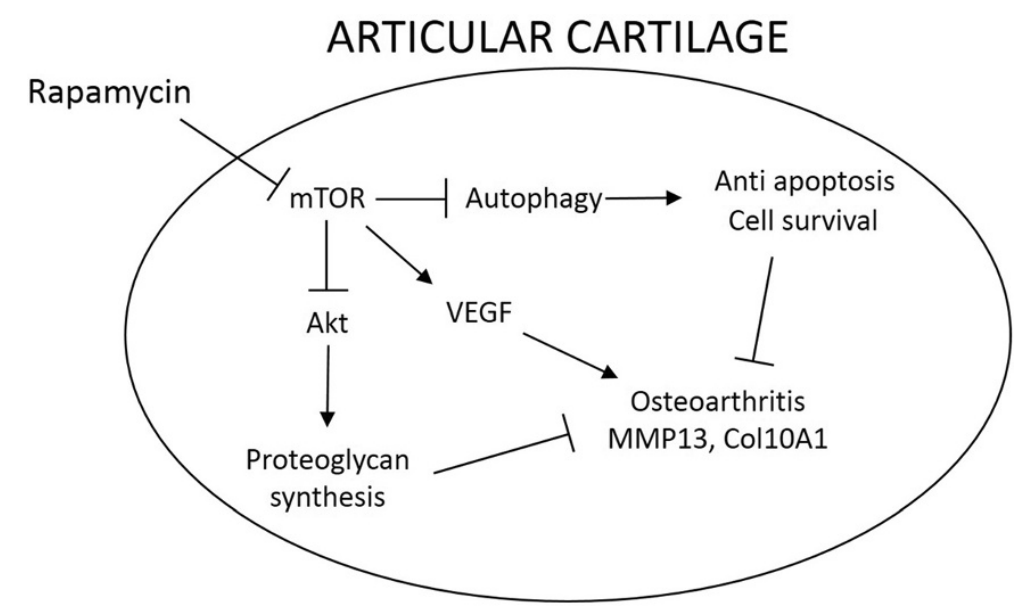

Figure 6 Proposed schematic model showing how rapamycin modulates osteoarthritis (OA) in our animal model. There are three potential mechanisms by which rapamycin has a beneficial effect on OA (autophagy, vascular endothelial growth factor (VEGF), and the Akt pathway). mTOR, mammalian target of rapamycin; MMP13, matrix metallopeptidase 13; COL10A1, collagen, type X alpha 1.

rapamycin regulated a change in the expression of OArelated genes through the modulation of apoptosis and reactive oxygen species (ROS) in human chondrocytes [8]. Similarly, during the development of OA, autophagy increased as an adaptive response to protect the cells from various stresses, while in severely damaged articular cartilage, autophagy was reduced [8]. The present study demonstrated that LC3-positive cells resided in healthy articular cartilage maintaining proteoglycan staining, and LC3-expressing cells decreased in degenerating articular cartilage after DMM surgery. Thus, it is plausible that articular cartilage degradation after surgical induction of OA is partially due to insufficient autophagy. In support of this hypothesis, our results also indicate that the beneficial effect of local intra-articular rapamycin treatment on OA-induced cartilage damage correlates with an increase in LC3-expressing cells (Figure 6).

Subsequently, we focused on the potential role of angiogenesis in modulating articular cartilage degeneration after OA and whether the beneficial effect of rapamycin can be explained by modulation of angiogenesis. Accumulating evidence indicates that joints affected by OA contain increased levels of VEGF in their articular cartilage and synovial fluid as well as several cytokines that stimulate VEGF production $[16,22,23]$. In a recent publication we have demonstrated that the intra-articular injection of stem cells transduced with sFLT1, an antagonist of VEGF, could reduce angiogenesis and improve the regeneration of articular cartilage without osteophyte development in a rat model of OA [24]. Interestingly, mTOR activation stimulates VEGF $[25,26]$, whereas rapamycin treatment reduces VEGF expression, which leads to the suppression of endothelial cell proliferation, survival, and migration [27]. In the current study, local intra-articular injection of rapamycin decreased the expression of VEGF after DMM when compared with the DMSO-treated mice, suggesting that decreased VEGF expression might play a role in the beneficial effect of rapamycin on articular cartilage after OA (Figure 6).

To further examine the beneficial effect of rapamycin on articular cartilage after $\mathrm{OA}$, we investigated chondrocyte hypertrophy. Chondrocyte hypertrophic-like changes also contribute to the progression of early and late stages of OA [28]. The induction of hypertrophic-like changes in healthy human chondrocytes causes calcification of the extracellular matrix $[29,30]$. Recently, hypertrophic differentiation of chondrocytes has also been reported to promote angiogenesis [31]; hence, the inhibition of chondrocyte hypertrophic-like alterations could be a therapeutic target to block the progression of OA [28]. mTOR is associated with the development of hypertrophic changes [32-34], and mTOR inhibition by rapamycin decreases chondrocyte hypertrophy in the growth plate $[35,36]$. The expression of COL10A1 and MMP13 are the most widely used markers for identifying hypertrophic chondrocytes [37-39]. In the current study, rapamycin treatment was found to reduce the expression of COL10A1 and MMP13 in comparison to mice treated with DMSO after DMM surgery. However, in the rapamycin-treated mice, the expression of COL10A1 and MMP13 at 12 weeks were increased compared to those at 8 weeks. These findings suggest that the intra-articular injection of rapamycin reduced the hypertrophic changes in the articular cartilage in this experimental model of OA, which contributed to a delay in OA progression.

There are some limitations in this study. First, the optimal dosage and frequency of rapamycin might be different in mice and humans, although the results suggest that local intra-articular injection of rapamycin delayed articular cartilage degradation. We also need to examine 
the effect of intra-articular injection of rapamycin at a lower dosage and frequency in larger animal models, before we apply the injection of rapamycin in a clinical setting. Second, experimental OA was induced by DMM. Lesions in the DMM model progressed from mild to moderate OA compared to the anterior cruciate ligament transection model. Therefore, experimental OA induced by DMM differs from the primary OA observed in humans. In addition, young mice were used in this study, and although young mice have been widely used for this type of study, the regenerative capacity in young animals is most likely superior to that of aged animals, which could obscure the process of cartilage degeneration. In future studies, aged and large animal models should be utilized to test the effects of rapamycin treatment on articular cartilage in primary OA.

\section{Conclusion}

In conclusion, our results demonstrated that the intraarticular injection of rapamycin reduce mTOR expression, which leads to a delay in cartilage degradation after surgically inducing joint instability. Activation of LC3 (an autophagy marker) in the OA-induced chondrocytes as well as reduction in VEGF, COL10A1, and MMP13 expression was also observed in the rapamycin-treated knees. Our observations suggest that local intra-articular injection of rapamycin may represent a strategy to prevent the development of articular cartilage damage, while limiting the side effect of systemic delivery of rapamycin. Further studies that explore mTOR inhibition will provide novel insights into the pathophysiology of $\mathrm{OA}$ and could lead to the establishment of new therapeutic approaches for slowing the progression of OA.

\section{Abbreviations \\ COL10A1: collagen, type $X$ alpha 1; DMM: destabilization of the medial meniscus; DMSO: dissolved in dimethyl sulphoxide; ECM: extracellular matrix; ER: endoplasmic reticulum; LC3: light chain 3; MMP13: matrix metallopeptidase 13; mTOR: mammalian target of rapamycin; OA: osteoarthritis; PBS: phosphate- buffered saline; PI-3 kinase: phosphoinositide 3 kinase; p-mTOR: phospho- mammalian target of rapamycin; qPCR: quantitative RT-PCR; ROS: reactive oxygen species; VEGF: vascular endothelial growth factor.}

\section{Competing interests}

The authors declare that they have no competing interests.

\section{Authors' contributions}

KT and YK conceived of the study, secured funding, carried out the animal model studies, participated in its design and coordination and the interpretation of the data, performed the statistical analysis, and drafted the manuscript. MKo and NG carried out the animal model studies and participated in the interpretation of the data. JHC, TM, RK, MKu and FF participated in the interpretation of the data. JH secured funding, participated in the design and coordination of the study and the interpretation of the data, and corrected/approve the final draft of the manuscript. All authors were involved in drafting the article or revising it critically for important intellectual content. All authors have read and approved the final manuscript.

\section{Acknowledgements}

The authors are grateful for the technical advice provided by Ms Jessica Tebbets, and the editorial assistance of Mrs Bria King. This study was funded in part by a grant awarded to Johnny Huard from the Department of Defense (W81WXH-08-0076) and the Henry J Mankin Endowed Chair at the University of Pittsburgh.

\section{Author details}

'Stem Cell Research Center, University of Pittsburgh, Pittsburgh, PA 15219, USA. ${ }^{2}$ Department of Orthopaedic Surgery, University of Pittsburgh, Pittsburgh, PA 15260, USA. ${ }^{3}$ Department of Orthopaedic Surgery, Kobe University Graduate School of Medicine, Kobe 650-0017, Japan. ${ }^{4}$ Stem Cell Research Center, Department of Orthopaedic Surgery, University of Pittsburgh, School of medicine, 450 Technology Drive, Bridgeside Point II, Suite 206, Pittsburgh, PA 15219, USA.

Received: 27 April 2014 Accepted: 5 November 2014 Published online: 17 November 2014

\section{References}

1. Vignon E, Arlot M, Meunier P, Vignon G: Quantitative histological changes in osteoarthritic hip cartilage. Morphometric analysis of 29 osteoarthritic and 26 normal human femoral heads. Clin Orthop Relat Res 1974, 103:269-278.

2. Kronenberg HM: Developmental regulation of the growth plate. Nature 2003, 423:332-336.

3. Levine B, Klionsky DJ: Development by self-digestion: molecular mechanisms and biological functions of autophagy. Dev Cell 2004, 6:463-477.

4. Levine B, Kroemer G: Autophagy in the pathogenesis of disease. Cell 2008, 132:27-42.

5. Eskelinen EL, Saftig P: Autophagy: a lysosomal degradation pathway with a central role in health and disease. Biochim Biophys Acta 2009, 1793:664-673.

6. Mizushima N, Levine B, Cuervo AM, Klionsky DJ: Autophagy fights disease through cellular self-digestion. Nature 2008, 451:1069-1075.

7. Komatsu M, Ueno T, Waguri S, Uchiyama Y, Kominami E, Tanaka K: Constitutive autophagy: vital role in clearance of unfavorable proteins in neurons. Cell Death Differ 2007, 14:887-894.

8. Sasaki H, Takayama K, Matsushita T, Ishida K, Kubo S, Matsumoto T, Fujita N, Oka S, Kurosaka M, Kuroda R: Autophagy modulates osteoarthritis-related gene expression in human chondrocytes. Arthritis Rheum 2012, 64:1920-1928.

9. Carames B, Hasegawa A, Taniguchi N, Miyaki S, Blanco FJ, Lotz M: Autophagy activation by rapamycin reduces severity of experimental osteoarthritis. Ann Rheum Dis 2012, 71:575-581.

10. Koehl GE, Andrassy J, Guba M, Richter S, Kroemer A, Scherer MN, Steinbauer M, Graeb C, Schlitt HJ, Jauch KW, Geissler EK: Rapamycin protects allografts from rejection while simultaneously attacking tumors in immunosuppressed mice. Transplantation 2004, 77:1319-1326.

11. Hudes G, Carducci M, Tomczak P, Dutcher J, Figlin R, Kapoor A, Staroslawska E, Sosman J, McDermott D, Bodrogi I, Kovacevic Z, Lesovoy V, Schmidt-Wolf IG, Barbarash O, Gokmen E, O'Toole T, Lustgarten S, Moore L, Motzer RJ, Global ARCC, Global ARCC Trial: Temsirolimus, interferon alfa, or both for advanced renal-cell carcinoma. N Engl J Med 2007, 356:2271-2281.

12. Cravedi $P$, Ruggenenti $P$, Remuzzi $G$ : Sirolimus to replace calcineurin inhibitors? Too early yet. Lancet 2009, 373:1235-1236.

13. Glasson SS, Blanchet TJ, Morris EA: The surgical destabilization of the medial meniscus (DMM) model of osteoarthritis in the 129/SvEv mouse. Osteoarthritis Cartilage 2007, 15:1061-1069.

14. Carames B, Taniguchi N, Seino D, Blanco FJ, D'Lima D, Lotz M: Mechanical injury suppresses autophagy regulators and pharmacologic activation of autophagy results in chondroprotection. Arthritis Rheum 2012, 64:1182-1192.

15. Glasson SS, Chambers MG, Van Den Berg WB, Little CB: The OARSI histopathology initiative - recommendations for histological assessments of osteoarthritis in the mouse. Osteoarthritis Cartilage 2010, 18:S17-S23.

16. Wei T, Kulkarni NH, Zeng QQ, Helvering LM, Lin X, Lawrence F, Hale L, Chambers MG, Lin C, Harvey A, Ma YL, Cain RL, Oskins J, Carozza MA, Edmondson DD, Hu T, Miles RR, Ryan TP, Onyia JE, Mitchell PG: Analysis of early changes in the articular cartilage transcriptisome in the rat meniscal tear model of osteoarthritis: pathway comparisons with the rat anterior cruciate transection model and with human osteoarthritic cartilage. Osteoarthritis Cartilage 2010, 18:992-1000. 
17. Wan X, Harkavy B, Shen N, Grohar P, Helman LJ: Rapamycin induces feedback activation of Akt signaling through an IGF-1R-dependent mechanism. Oncogene 2007, 26:1932-1940.

18. Shi $X$, Shen B, Yang J, Kang P, Zhou Z, Pei F: In vivo kinematics comparison of fixed- and mobile-bearing total knee arthroplasty during deep knee bending motion. Knee Surg Sports Traumatol Arthrosc 2014, 22:1612-1618.

19. Yin W, Park Jl, Loeser RF: Oxidative stress inhibits insulin-like growth factor-I induction of chondrocyte proteoglycan synthesis through differential regulation of phosphatidylinositol 3-Kinase-Akt and MEK-ERK MAPK signaling pathways. J Biol Chem 2009, 284:31972-31981.

20. Cravero JD, Carlson CS, Im HJ, Yammani RR, Long D, Loeser RF: Increased expression of the Akt/PKB inhibitor TRB3 in osteoarthritic chondrocytes inhibits insulin-like growth factor 1-mediated cell survival and proteoglycan synthesis. Arthritis Rheum 2009, 60:492-500.

21. Starkman BG, Cravero JD, Delcarlo M, Loeser RF: IGF-I stimulation of proteoglycan synthesis by chondrocytes requires activation of the $\mathrm{PI}$ 3-kinase pathway but not ERK MAPK. The Biochemical journal 2005, 389:723-729.

22. Pesesse L, Sanchez C, Henrotin Y: Osteochondral plate angiogenesis: a new treatment target in osteoarthritis. Joint Bone Spine 2011, 78:144-149.

23. Lotz M: Osteoarthritis year 2011 in review: biology. Osteoarthritis Cartilage 2012, 20:192-196

24. Matsumoto T, Cooper GM, Gharaibeh B, Meszaros LB, Li G, Usas A, Fu FH, Huard J: Cartilage repair in a rat model of osteoarthritis through intraarticular transplantation of muscle-derived stem cells expressing bone morphogenetic protein 4 and soluble Flt-1. Arthritis Rheum 2009, 60:1390-1405.

25. Bjornsti MA, Houghton PJ: The TOR pathway: a target for cancer therapy. Nat Rev Cancer 2004, 4:335-348.

26. Hsu T, Adereth Y, Kose N, Dammai V: Endocytic function of von HippelLindau tumor suppressor protein regulates surface localization of fibroblast growth factor receptor 1 and cell motility. J Biol Chem 2006, 281:12069-12080.

27. Guba M, von Breitenbuch $P$, Steinbauer M, Koehl G, Flegel S, Hornung M, Bruns CJ, Zuelke C, Farkas S, Anthuber M, Jauch KW, Geissler EK: Rapamycin inhibits primary and metastatic tumor growth by antiangiogenesis: involvement of vascular endothelial growth factor. Nat Med 2002, 8:128-135.

28. van der Kraan PM, van den Berg WB: Chondrocyte hypertrophy and osteoarthritis: role in initiation and progression of cartilage degeneration? Osteoarthritis Cartilage 2012, 20:223-232.

29. Fuerst M, Bertrand J, Lammers L, Dreier R, Echtermeyer F, Nitschke Y, Rutsch F, Schafer FK, Niggemeyer O, Steinhagen J, Lohmann CH, Pap T, Rüther W: Calcification of articular cartilage in human osteoarthritis. Arthritis Rheum 2009, 60:2694-2703.

30. Fuerst M, Niggemeyer O, Lammers L, Schafer F, Lohmann C, Ruther W: Articular cartilage mineralization in osteoarthritis of the hip. BMC Musculoskelet Disord 2009, 10:166.

31. Pesesse L, Sanchez C, Delcour JP, Bellahcene A, Baudouin C, Msika P, Henrotin Y: Consequences of chondrocyte hypertrophy on osteoarthritic cartilage: potential effect on angiogenesis. Osteoarthritis Cartilage 2013, 21:1913-1923.

32. McMullen JR, Sherwood MC, Tarnavski O, Zhang L, Dorfman AL, Shioi T, Izumo S: Inhibition of mTOR signaling with rapamycin regresses established cardiac hypertrophy induced by pressure overload. Circulation 2004, 109:3050-3055.

33. Park $\mathrm{H}$, Erbay E, Nuzzi $P$, Chen J: Skeletal myocyte hypertrophy requires mTOR kinase activity and S6K1. Exp Cell Res 2005, 309:211-219.

34. Sakaguchi M, Isono M, Isshiki K, Sugimoto T, Koya D, Kashiwagi A: Inhibition of mTOR signaling with rapamycin attenuates renal hypertrophy in the early diabetic mice. Biochem Biophys Res Commun 2006, 340:296-301.

35. Alvarez-Garcia O, Carbajo-Perez E, Garcia E, Gil H, Molinos I, Rodriguez J, Ordonez FA, Santos F: Rapamycin retards growth and causes marked alterations in the growth plate of young rats. Pediatr Nephrol 2007, 22:954-961.

36. Alvarez-Garcia O, Garcia-Lopez E, Loredo V, Gil-Pena H, Rodriguez-Suarez J, Ordonez FA, Carbajo-Perez E, Santos F: Rapamycin induces growth retardation by disrupting angiogenesis in the growth plate. Kidney Int 2010, 78:561-568
37. Kirsch T, von der Mark K: Remodelling of collagen types I, II and X and calcification of human fetal cartilage. Bone Miner 1992, 18:107-117.

38. Nurminskaya M, Linsenmayer TF: Identification and characterization of up-regulated genes during chondrocyte hypertrophy. Dev Dyn 1996, 206:260-271.

39. Alvarez J, Balbin M, Santos F, Fernandez M, Ferrando S, Lopez JM: Different bone growth rates are associated with changes in the expression pattern of types II and X collagens and collagenase 3 in proximal growth plates of the rat tibia. J Bone Miner Res 2000, 15:82-94.

\section{doi:10.1186/s13075-014-0482-4}

Cite this article as: Takayama et al:: Local intra-articular injection of rapamycin delays articular cartilage degeneration in a murine model of osteoarthritis. Arthritis Research \& Therapy 2014 16:482.

\section{Submit your next manuscript to BioMed Central and take full advantage of:}

- Convenient online submission

- Thorough peer review

- No space constraints or color figure charges

- Immediate publication on acceptance

- Inclusion in PubMed, CAS, Scopus and Google Scholar

- Research which is freely available for redistribution 